\title{
Introducing the new BTS guideline: the investigation and management of pleural malignant mesothelioma
}

\author{
Ian Woolhouse, ${ }^{1}$ Nick A Maskell ${ }^{2}$
}

\section{RATIONALE FOR THE NEW GUIDELINE}

Malignant pleural mesothelioma (MPM) is an aggressive tumour with a poor prognosis. In 2012, 2535 mesothelioma deaths were reported in the UK, and the incidence is predicted to increase.

Only two-thirds of patients in England and Wales receive active anticancer treatment (chemotherapy, radiotherapy and surgery) for MPM and overall median survival is poor at 9.5 months, with 1 -year and 3 -year survival rates of only $41 \%$ and $12 \%$, respectively. ${ }^{1}$ The poor survival rates, taken together with the significant variation in treatment and outcomes across the UK, highlight the need for an evidence-based guideline to facilitate the highest standards of care for all patients with mesothelioma in the UK.

In 2007, the British Thoracic Society (BTS) statement on mesothelioma was published in response to a request from the National Health Executive in England. ${ }^{2}$ While very informative, this statement did not constitute an evidence-based review of all the literature and a number of key clinical trials have been published in the last 10 years. In particular, there have been a number of landmark randomised controlled trials (RCTs) to assess the effectiveness of surgical treatments and prophylactic radiotherapy in MPM as well as studies of new biomarkers and novel chemotherapeutic agents.

BTS established a mesothelioma guideline development group, co-chaired by Professor Nick Maskell and Dr Ian Woolhouse, in 2014, tasked with producing an evidence-based guideline to assist with the investigation and management of pleural malignant mesothelioma. These guidelines are published as a supplement in this issue of Thorax. ${ }^{3}$ A summary of recommendations and good practice points is available in BMJ Open Respiratory Research. ${ }^{4}$

\footnotetext{
'Department of Respiratory Medicine, University Hospitals Birmingham NHS Foundation Trust, Birmingham, UK

${ }^{2}$ Academic Respiratory Unit, Southmead Hospital, University of Bristol, Bristol, UK
}

Correspondence to Prof Nick A Maskell, Academic Respiratory Unit, University of Bristol, Bristol, BS10 5NB, UK; nick.maskell@bristol.ac.uk

\section{METHODOLOGY}

The evidence review methodology and grading of recommendations follows the National Institute for Health and Care Excellence accredited BTS guideline production process, which is based on Scottish Intercollegiate Guidelines Network methodology and adheres to the Appraisal of Guidelines for Research and Evaluation instrument. ${ }^{5}$

\section{RECOMMENDATIONS}

Recommendations are made relating to key points in the patient pathway, from optimal diagnostic strategies for suspected MPM, symptom control, anticancer treatment, follow-up and organisation of services. A number of key areas are summarised below.

\section{Biomarkers}

The literature search revealed a large volume of evidence exploring different biomarkers that may have a role in MPM. Several markers such as mesothelin, fibulin-3, osteopontin and megakaryocyte potentiating factor have been extensively studied internationally. There is no diagnostic biomarker which is able to consistently diagnose MPM with a sensitivity and specificity above 90\%. Mesothelin and osteopontin have a relatively high specificity in the diagnosis of MPM, but fibulin-3 shows variable performance (sensitivity range 22\%-100\%). ${ }^{6}$ The guideline recommends that biomarkers should not be offered in isolation as a diagnostic test in MPM. However, in patients not fit enough for more invasive tests who have suspicious cytology and consistent radiology, a raised mesothelin can assist a mesothelioma multidisciplinary team in reaching a clinico-radio-cytological diagnosis. Further research is needed before biomarkers can be used routinely to predict the response to treatment or survival.

\section{Surgical intervention}

The MesoVATS study compared video-assisted thoracoscopic (VATS) partial pleurectomy to talc (poudrage or slurry). ${ }^{78}$ VATS pleurectomy was not associated with survival benefit (primary outcome) nor benefits to lung function. Patients who underwent VATS partial pleurectomy had a significantly higher complication rate and longer hospital stay. The Mesothelioma and Radical Surgery (MARS) feasibility study assessed extrapleural pneumonectomy (EPP) versus no EPP for patients with MPM. ${ }^{9}$ Median survival was 14.4 months (5.3-18.7) for the EPP group and 19.5 months (13.4 to time not yet reached) for the no EPP group. There has been much discussion around the validity of the MARS trial results. In particular, criticism that the study was not powered to detect a survival advantage attributable to EPP and that the operative mortality was higher than that of other contemporary series. The MARS trial authors have subsequently responded that the EPP mortality in MARS (2 of 19; $10.5 \%$; 95\% confidence limits $1.3 \%$ to $33.1 \%$ ) lies within the range reported in a systematic review of 34 studies, including 2320 patients, where 30-day mortality ranged from $0 \%$ to $11.8 \% .^{9}$ Furthermore, the authors note that the median survival of patients in the EPP arm of MARS of 14.4 months from randomisation is in keeping with major series in the literature that report median survival times of 10 to 14 months.

Based on these two landmark surgical trials, the guideline does not recommend the use of VATS partial pleurectomy for fluid control in MPM or the use of EPP for disease control in MPM. The role of pleurectomy decortication remains controversial and the guideline recommends that potentially suitable patients are offered entry into the MARS-2 trial (NCT02040272).

\section{Systemic anticancer treatment \\ First line}

Systemic therapy is the only proven intervention to improve survival in mesothelioma. The mesothelioma avastin cisplatin pemetrexed study (MAPS) was a randomised, controlled, open-label, phase III trial. ${ }^{10}$ Patients with MPM were randomised to receive pemetrexed plus cisplatin with or without bevacizumab. Overall survival was significantly longer in the bevacizumab group (median 18.8 months vs 16.1 months; $\mathrm{P}=0.0167$ ). The rate of adverse events was similar in the two groups, although higher rates of hypertension and more thrombotic events were noted in the bevacizumab group. Bevacizumab treatment is not currently licensed for use in the UK and is therefore not available in the National Health 
Service. On this basis, the guideline recommends that patients with MPM with good performance status are offered first-line therapy with cisplatin and pemetrexed, but notes that the addition of bevacizumab to this regime can prolong survival further. Recently, a phase II placebo-controlled double-blind trial showed an improvement in progression-free survival (PFS) associated with addition of nintedanib (a tyrosine kinase inhibitor) to chemotherapy with subgroup analysis suggesting possible efficacy in the epithelioid subtype. This trial showed a median PFS of 9.4 months versus 5.7 months $(\mathrm{P}=0.01) .{ }^{11}$ The global LUME Meso phase III trial is currently enrolling (NCT01907100) in epithelioid mesothelioma.

Sarcomatoid mesothelioma represents the most aggressive subtype with frequent loss of the enzyme ASS1 rendering them susceptible to arginine deprivation therapy. Following a phase I study, ATOMIC-meso (NCT02709512) is studying the effect of giving ADI-PEG20 with pemetrexed/ cisplatin chemotherapy in patients with non-epithelioid mesothelioma.

\section{Relapsed setting}

There currently remains no standard of care for patients with relapsed mesothelioma. Pembrolizumab is an antibody-based therapeutic agent that is targeted at the immune inhibitory protein, programmed cell death 1 (PD1). This protein engages with and inhibits T-cell-mediated immunity against cancers. By interacting with PD1, pembrolizumab reactivates the immune system by essentially removing the tumour's camouflage. This leads to the immune system attacking the cancer.

The Keynote 28 study investigators (NCT02054806) have reported that pembrolizumab, in patients with PDL1-positive mesothelioma, has clinically significant activity associated with a $20 \%$ response rate with responses lasting, on average, a year, and $52 \%$ patients attain stable disease. Critically, the expression of the PDL1 (programmed death 1 ligand), a potential predictive biomarker for pembrolizumab, was not shown to be associated with efficacy, implying that patients could benefit irrespective of the biomarker.

While this is exciting, there is not enough published data to inform this set of BTS guidelines, and we await future published research in this area with interest.

Randomised trials evaluating antiPD1 monotherapy will provide vital information regarding the magnitude of safety and efficacy, and in this regard the CONFIRM (NCT03063450) and PROMISE (NCT02991482) trials are currently enrolling.

Combination immunotherapy may be an effective approach based on promising efficacy reported in the MAPS2 trial evaluating nivolumab and ipilimumab. Accordingly, CheckMate 743 is a phase III RCT of nivolumab in combination with ipilimumab versus pemetrexed/cisplatin in the first-line setting (NCT02899299).

\section{Radiotherapy}

The SMART trial was a randomised, multicentre, phase III trial evaluating whether prophylactic radiotherapy reduces the incidence of procedure tract metastases (PTM) after surgical and largebore pleural procedures. ${ }^{12}$ The authors found no difference between the PTM rates of the immediate and deferred radiotherapy groups $(9 / 102$ vs $16 / 101$; $\mathrm{P}=0.14$ ). This data in combination with the results of several other smaller studies led the guideline committee to not recommend offering prophylactic radiotherapy to chest wall procedure tracts routinely. The PIT trial (ISRCTN04240319) is due to be published shortly and will add valuable information to this field. The authors of the PIT and SMART trials are also planning to perform a meta-analysis of both studies.

With regards to radiotherapy for symptom palliation, the guideline states that it should be considered in cases where localised pain matches areas of underlying disease. SYSTEMS2 (ISRCTN12698107) is a randomised trial currently recruiting in the UK. It is comparing standard (20Gy/5 f) versus escalated dose (36Gy/6f) radiotherapy for pain control, and the results will inform practice.

\section{FUTURE RESEARCH}

Guidelines can only appraise the evidence of published trials in peer-reviewed journals. However, it is recognised that this is an extremely exciting time for mesothelioma research with a range of important oncological trials under way and some starting to present results at international conferences.

We hope colleagues will continue to support these trials and encourage patient participation. A useful link for regular trial updates can be found online (http://www. mesothelioma.uk.com/information-andsupport/clinical-trials). ${ }^{13}$

\section{SUMMARY}

Following the publication of these new guidelines, the imminent challenge is to implement the key recommendations to ensure patients receive the best possible evidence-based care. We hope these guidelines will provide a platform for future research and quality improvement.

Contributors IW and NAM were the lead authors with overall responsibility for the article.

Competing interests None declared.

Provenance and peer review Not commissioned; externally peer reviewed.

(c) Article author(s) (or their employer(s) unless otherwise stated in the text of the article) 2018. All rights reserved. No commercial use is permitted unless otherwise expressly granted.

\section{A) Check for updates}

To cite Woolhouse I, Maskell NA. Thorax 2018:73:210-212.

Received 14 December 2017

Revised 12 January 2018

Accepted 22 January 2018

Thorax 2018;73:210-212.

doi:10.1136/thoraxinl-2017-211416

\section{REFERENCES}

1 National Lung Cancer Audit. Pleural Mesothelioma Report 2016. London, UK: Royal College of Physicians, 2016.

2 British Thoracic Society Standards of Care Committee. BTS statement on malignant mesothelioma in the UK, 2007. Thorax 2007;62(Suppl 2):ii1-ii19.

3 Woolhouse I, Bishop L, Darlison L, et al. BTS Guideline for the investigation and management of malignant pleural mesothelioma. Thorax 2018.

4 Woolhouse I, Bishop L, Darlison L, et al. BTS Guideline for the investigation and management of malignant pleural mesothelioma. BMJ Open Respiratory Research 2018.

5 AGREE Advancing the science of practice guidelines. AGREE II. www.agreetrust.org/resource-centre/agreeii/

6 Creaney J, Dick IM, Meniawy TM, et al. Comparison of fibulin-3 and mesothelin as markers in malignant mesothelioma. Thorax 2014;69:895-902.

7 Pass HI, Levin SM, Harbut MR, et al. Fibulin-3 as a blood and effusion biomarker for pleural mesothelioma. N Engl J Med 2012;367:1417-27.

8 Rintoul RC, Ritchie AJ, Edwards JG, et al. Efficacy and cost of video-assisted thoracoscopic partia pleurectomy versus talc pleurodesis in patients with malignant pleural mesothelioma (MesoVATS) an open-label, randomised, controlled trial. Lancet 2014;384:1118-27.

9 Lim E. 195 A feasibility study comparing (extended) pleurectomy decortication versus no pleurectomy decortication in the multimodality management of patients with malignant pleural mesothelioma: the MARS 2 study. Lung Cancer 2016;91(Suppl 1):S71.

10 Zalcman G, Mazieres J, Margery J, et al. Bevacizumab $15 \mathrm{mg} / \mathrm{kg}$ plus cisplatin-pemetrexed (CP) triplet versus $\mathrm{CP}$ doublet in malignant pleural mesothelioma (MPM): results of the IFCT-GFPC-0701 MAPS randomized phase 3 trial. J Clin Oncol 2015;33:7500. 
11 Grosso F, Steele N, Novello S, et al. Nintedanib plus pemetrexed/cisplatin in patients with malignant pleural mesothelioma: phase II results from the randomized, placebo controlled LUME-Meso trial. J Clin Oncol 2017;35:3591-600.
12 Clive AO, Taylor H, Dobson L, et al. Prophylactic radiotherapy for the prevention of procedure-tract metastases after surgical and large-bore pleural procedures in malignant pleural mesothelioma (SMART): a multicentre, open-label, phase
3 , randomised controlled trial. Lancet Oncol 2016;17:1094-104.

13 Mesothelioma UK. Clinical Trials. http://www. mesothelioma.uk.com/information-and-support/ clinical-trials 